\title{
A PROTOTYPE CLIMBING ROBOT FOR INSPECTION OF COMPLEX FERROUS STRUCTURES
}

\author{
G. PETERS, D. PAGANO, D.K. LIU \\ ARC Centre of Excellence for Autonomous Systems, University of Technology, Sydney \\ Australia, POBox 123, Broadway, NSW 2007, \{Gregory.Peters@uts.edu.au, \\ David.Pagano@student.uts.edu.au, dkliu@eng.uts.edu.au\} \\ K. WALDRON \\ kwaldron@stanford.edu
}

\begin{abstract}
This is where the abstract should be placed. It should consist of one paragraph giving a concise summary of the material in the article below. Replace the title, authors, and addresses with your own title, authors, and addresses. You may have as many authors and addresses as you like. It is preferable not to use footnotes in the abstract or the title; the acknowledgments of funding bodies etc. are to be placed in a separate section at the end of the text.
\end{abstract}

\section{Introduction}

\subsection{Motivation and Requirements}

The RVC project aims to design and develop an automated, modular robot that is capable of climbing highly complex ferrous structures, such as bridges, while performing inspections for rust, paint degradation and maintenance needs. This periodical inspection is a vital part of prolonging the life of the structure and ensuring public safety as well as saving on maintenance costs. The obvious height dangers as well as the constant presence of vehicles present a significant danger both to bridge workers and bridge users during maintenance and inspection work.

Highly complex structures such as bridges have irregular geometry that makes exploration difficult. Furthermore the surface is typically unsmooth, with many large rivets, bolts and flanges. A highly reliable adhesion mechanism is also required that must be failsafe and not detach in the event of power failure. The challenge of the RVC project is the development of a small proof of concept prototype robot to meet these requirements. 


\subsection{State of the Art}

Significant attention has been provided to climbing on relatively smooth surfaces. The adhesion and locomotion methods have been the major two issues and received much discussion [1] [2]. Adhesion methods can be divided into four main principles, namely, suction force, gripping force, magnetic force and other adhesion technologies.

Pneumatic adhesion, which requires the production of a pressure differential between the robot and the surrounding air, has been the preferred method for many robots, because of its ability to work on many surface types. These systems may use passive suction cups [3] [4] [5], however do not perform well on nonsmooth surfaces, alternatively vacuum or vortex systems may be applied [6] to work with slightly rougher surfaces although do not retain adhesion during power failure. Excessively bulky and power intensive equipment is required to deal with very rough surfaces.

Magnetic adhesion using permanent magnets or electromagnets works only in specific environments where the surface is ferrous. Electromagnetic systems are typically much lighter than permanent magnet systems and provide simple adhesion control, however do not retain adhesion during power failure. Permanent magnet based systems have been coupled with tracks [7], wheels [8] or complex shunting systems [9] to facilitate low force detachment while retaining failsafe adhesion. The concept of compliantly distributed adhesion [10] simplifies the detachment process while providing significant adaptation to surface irregularities.

Locomotion of climbing robots through highly complex environments has been found to be difficult without the use of legs. Systems based on four or more legs provide stability and redundant support, however, significantly increases the control complexity, system cost, power consumption and weight [2].

*** Literature Review must include some information about control methods from David $* * *$

\section{Modular design of the Robot}

For robots that climb highly complex environments the key challenge is to maximise locomotion ability and the exploration workspace. This should be done while retaining a non task-specific platform to increase the diversity of applications the platform can be applied to, reducing development costs. 
*** Discuss reason for modular architecture in mechanical design $* * *$

\subsection{Mechanical Design}

*** Introduce mechanical design of robot $* * *$

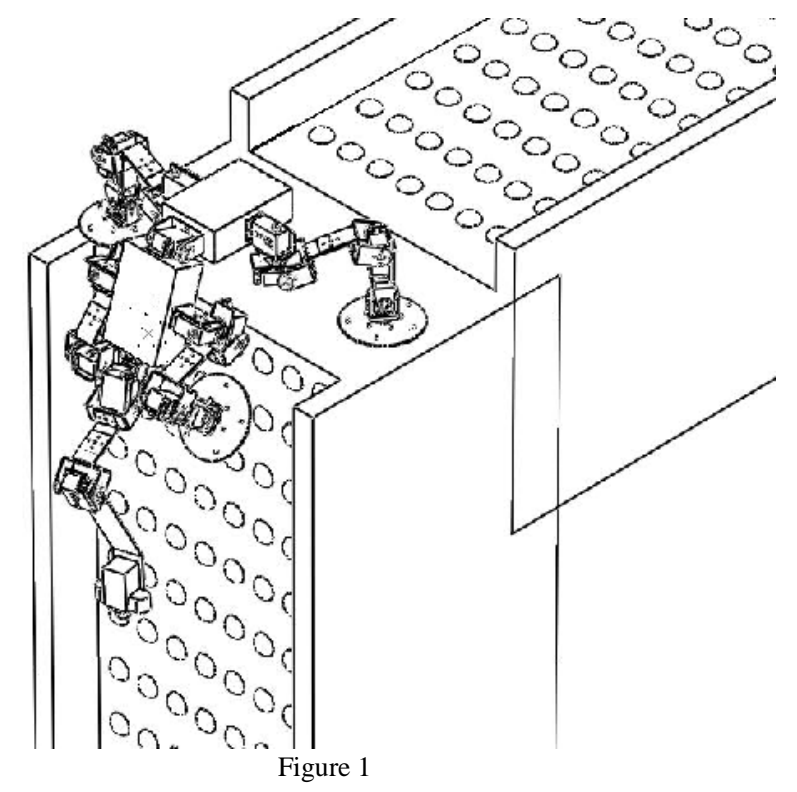

\subsection{Attachment Mechanism}

*** Introduce the attachment mechanism ***

\section{Control System}

*** DAVIDS SECTION -->DK comment: It is important here to focus on why and how we have implemented whatever method we have chosen as we have not made any novel control method, just a way of using what is available. This means this section will not need to be too long or detailed ***

\subsection{Kinematics}

The control system for the robot has been developed based on a hierarchy model. 
*** Introduce the gait control and kinematics control system ***

As each walking module features a pair of 3-DOF legs which feature standard kinematics, and the number of walking modules is expandable, the leg control system has also been designed in a modular nature. The robot is controlled via a Matlab Simulink based motion model. A comparative analysis between Cartesian space and Virtual Force Field control, both methods provided zero steady state error however Virtual Force Field control was selected because of its fast settling time.

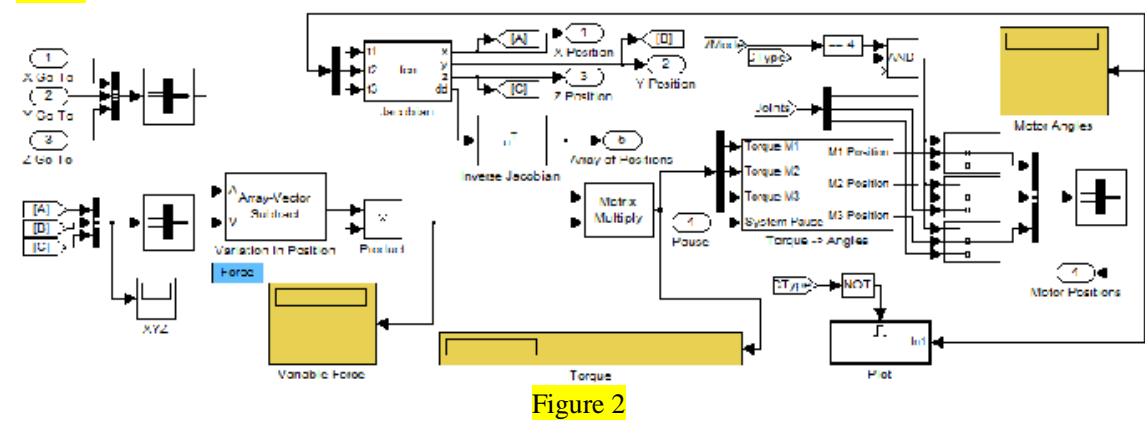

The kinematics and gait control will be moved onboard the robot during subsequent work.

\subsection{Onboard Control}

***Greg: Introduce the onboard low level control ***

The low level onboard control layer for each walking module has been based around an Atmega128-16AU microcontroller. Each module communicates to the master high level controller via a modularly expandable Inter-Integrated Circuit $\left(\mathrm{I}^{2} \mathrm{C}\right)$ communications bus. The data is sent via a custom communications protocol that has been developed to allow future modular expansion of the robot. Each of the six motors per module is controlled via the Hitec Multi-protocol Interface (HMI), which utilises a one wire bidirectional serial interface. This facilitates motor position, voltage and current feedback as well as position and speed control.

\section{Experimental Evaluation}

*** Here we will discuss the implementation and any results, may need to generate some more results or tables (ie time taken to climb vs other robots, or 
load support, or transition time). This is also the place to discuss any issues we came across (but not too many). For example 'the construction of the prototype allowed us to analyze joint lengths, and climbing ability' ***

\section{Conclusion}

*** Here we will finish and discuss the future work, stressing that this is far from a complete project $* * *$

\section{References}

1. Longo, D. \& Muscato, G. 2008, 'Adhesion techniques for climbing robots: State of the art and experimental considerations', paper presented to the 11th Internation Conference on Climbing and Walking Robots and the Support Technologies for Mobile Machines, Coimbra, Portugal, 11/11/2008.

2. Silva, M. \& Machado, J. 2008, 'Climbing robots: A survey of technologies and applications', CLAWAR 2008, Coimbra, Portugal, pp. 1133-1140.

3. Zhu, J., Sun, D. \& Tso, S.-K. 2002, 'Development of a Tracked Climbing Robot', Journal of Intelligent and Robotic Systems, no. 35, pp. 427-444.

4. Balaguer, C., Gimenez, A. \& Jardon, A. 2005, 'Climbing Robots' Mobility for Inspection and Maintenance of 3D Complex Environments', Autonomous Robots, vol. 18, no. 2 - March 2005, pp. 157-169.

5. Brockmann, W. \& Mösch, F. 2005, 'Climbing Without a Vacuum Pump', in, Climbing and Walking Robots, Springer, Berlin Heidelberg, pp. 935-942.

6. Hillenbrand, C., Schmidt, D. \& Berns, K. 2008, 'CROMSCI - A Climbing Robot With Multiple Sucking Chambers For Inspections Tasks', WSPC, Robotics Research Lab, Department of Computer Science, University of Kaiserslautern, Germany.

7. Shen, W., Gu, J. \& Shen, Y. 2005, 'Proposed Wall Climbing Robot with Permanent Magnetic Tracks for Inspecting Oil Tanks', IEEE International Conference on Mechantronics \& Automation, IEEE, Niagra Falls, Canada.

8. Tache, F., Fischer, W., Caprari, G., Siegwart, R., Moser, R. \& Monfafa, F. 2009, 'Magnetbike: A magnetic Wheeled Robot With High Mobility for Inspecting Complex Shaped Structures', unpublished, Autonomous Systems Lab, Eidgen"ossische Technische Hochschule Z"urich (ETHZ)

9. Hirose, S., Imazato, M., Kudo, Y. \& Umetani, Y. 1986, 'Internally-Balanced Magnetic Unit', Advanced Robotics, vol. 3, no. 1, pp. 225-242.

10. Berengueres, J., Tadakuma, K., Kamoi, T. \& Kratz, R. 2007, 'Complient Distributed Magnetic Adhesion Device for Wall Climbing', 2007 IEEE International Conference on Robotics and Automation, IEEE, Roma, Italy. 11. 
